\title{
Maintenance of ATP concentrations in and of fertilizing ability of fowl and turkey spermatozoa in vitro
}

\author{
G. J. Wishart \\ Department of Reproductive Physiology, Agricultural Research Council's Poultry Research Centre, \\ Roslin, Midlothian EH25 9PS, U.K.
}

\begin{abstract}
Summary. Fowl and turkey spermatozoa, when diluted in a glutamate-based medium and incubated at $40^{\circ} \mathrm{C}$ under aerobic conditions showed a similar rate of oxygen utilization and maintained similar cellular ATP concentrations. Under anaerobic conditions, the glycolytic metabolism of fowl spermatozoa was sufficient to maintain a high ATP concentration, whilst turkey spermatozoa had a much lower glycolytic ability and their ATP levels fell rapidly. The maintenance of high sperm ATP concentrations was directly related to subsequent fertilizing ability of the spermatozoa. Diluted fowl semen showed good fertilizing ability $(>70 \%)$ under aerobic conditions and under anaerobic conditions in the presence of $10 \mathrm{~mm}$-glucose, but diluted turkey semen maintained good fertilizing ability only under aerobic conditions.
\end{abstract}

\section{Introduction}

Fowl and turkey spermatozoa differ metabolically in that turkey spermatozoa have a lower glycolytic ability (McIndoe \& Lake, 1973; Sexton, 1974). However, despite the disparity in the time for which it is practicable to hold semen of these species at low temperatures above $0^{\circ} \mathrm{C}$ before artificial insemination, whilst maintaining fertility in excess of $90 \%$ (fowl, $24 \mathrm{~h}$ : Lake \& Ravie, 1979; turkey, $5 \mathrm{~h}$ : Lake \& Stewart, 1978), these metabolic differences have not been related to the energy status and fertilizing ability of the spermatozoa.

The present work correlates oxidative and glycolytic capacity with maintenance of ATP levels in and with fertilizing ability of fowl and turkey spermatozoa incubated in vitro at $40^{\circ} \mathrm{C}$.

\section{Materials and Methods}

\section{Animals and semen collection}

The fowl hens were a commercial layer type and the semen donors were from a layer-type control strain obtained from Ross Poultry Ltd, Newbridge, Midlothian. The turkeys were of a medium-weight type obtained from British United Turkeys Ltd, Cheshire, England. All birds were kept in $14 \mathrm{~h}$ light/24 $\mathrm{h}$ and fed commercial breeders ration ad libitum.

The birds were kept in cages to allow collection of eggs from individual hens. Semen free from transparent fluid was collected from fowls (Lake, 1957) and turkeys (Lake \& Stewart, 1978). Semen samples from 12-14 birds were pooled and were transported to the laboratory within $10 \mathrm{~min}$ (fowl) or $20 \mathrm{~min}$ (turkey) of collection.

Numbers of spermatozoa were estimated using a Coulter counter (Lake \& Ravie, 1979) or by relating DNA content (Burton, 1956) of samples to numbers of spermatozoa. 


\section{Incubation medium}

In the absence of information on the micro-environment of spermatozoa in the utero-vaginal sperm-host glands of the female reproductive tract, an incubation medium with the ionic constituents of fowl seminal plasma was chosen. The medium contained $1.0 \mathrm{~mm}-\mathrm{KH}_{2} \mathrm{PO}_{4} ; 10 \mathrm{mM}-\mathrm{KCl} ; 5$ $\mathrm{mM}-\mathrm{MgCl}_{2} ; 20 \mathrm{mM}-\mathrm{NaCl} ; 32.5 \mathrm{mM}-\mathrm{NaOH} ; 100 \mathrm{~mm}$-sodium glutamate and $67.5 \mathrm{mM}-N$, $N$-bis(2hydroxyethyl)-2-aminoethane sulphonic acid. In this medium, concentrations of $\mathrm{PO}_{4}{ }^{3-}, \mathrm{Cl}^{-}, \mathrm{Na}^{+}$, $\mathrm{K}^{+}$and $\mathrm{Mg}^{2+}$ were as reported by Lake (1971) for fowl seminal plasma. Glutamate concentrations were related to the value of $106 \pm 12 \mathrm{~mm}$ (mean \pm s.d. for 5 determinations of pooled samples) for fowl seminal plasma glutamate (unpublished results). The osmolarity of this medium was 375 $\mathrm{mosmol} / \mathrm{kg} \mathrm{H} \mathrm{H}_{2} \mathrm{O}$ and the $\mathrm{pH}$ was $7 \cdot 4$.

Unwashed semen was diluted in this medium, 4-fold for fowl and 6-fold for turkey semen, to give approximately equal sperm concentrations. Aerobic incubations were performed with $3 \mathrm{ml}$ aliquots of diluted semen, shaken in $25 \mathrm{ml}$ flasks in a water bath at $40^{\circ} \mathrm{C}$. Anaerobic incubations were carried out in sealed $2 \mathrm{ml}$ tubes incubated under similar conditions. Aliquots of the same pooled semen sample were used for the measurement of at least 2 metabolic parameters under at least two different experimental conditions.

\section{Assay of metabolic parameters}

Oxygen consumption was measured with a Clark-type oxygen electrode. Standardization of oxygen content of samples was performed by the method of Robinson \& Cooper (1970). Lactate and glucose concentrations were measured in $\mathrm{KHCO}_{3}$-neutralized $\mathrm{HClO}_{4}$ extracts of diluted semen by the methods of Gutmann \& Wahlefeld (1974) and Huggett \& Nixon (1957) respectively; in the latter method 4-aminophenazone was replaced by $o$-dianisidine.

ATP was measured by the luciferase method (Strehler, 1974) in extracts made by plunging 0.2 $\mathrm{ml}$ aliquots of the incubation mixtures or of semen 'immediately' (3-4 sec) after ejaculation into an equal or greater volume of vigorously boiling water.

ADP in these extracts was measured as ATP after its conversion in a medium containing 0.05 M-glycine pH 9.0 with $0.5 \mathrm{~mm}$ - $\mathrm{MgSO}_{4}, 7.5 \mathrm{~mm}$-creatine phosphate and $14 \mathrm{U}$ creatine phosphokinase $/ \mathrm{ml}$, and incubation for $10 \mathrm{~min}$ at $40^{\circ} \mathrm{C}$.

AMP was also measured as ATP by converting initially to ADP with myokinase and then to ATP with phosphoenol-pyruvate and pyruvate kinase. To aid completion of these reactions, the pyruvate formed was reduced with lactate dehydrogenase. The AMP $\rightarrow$ ATP conversion was achieved by incubation of boiled semen extracts for $20 \mathrm{~min}$ at $40^{\circ} \mathrm{C}$ in a medium containing: $50 \mathrm{~mm}$ glycine $\mathrm{pH} 7.5,0.5 \mathrm{~mm}-\mathrm{Mg}_{2} \mathrm{SO}_{4}, 2.5 \mathrm{~mm}$-phosphoenol-pyruvate, $0.5 \mathrm{~mm}$-NADH, 25 units pyruvate kinase $/ \mathrm{ml}, 25$ units lactate dehydrogenase $/ \mathrm{ml}$ and 25 units myokinase $/ \mathrm{ml}$ and was incubated for 20 $\min$ at $40^{\circ} \mathrm{C}$.

Substrates, enzymes and other chemicals were from Sigma London Chemical Co. Ltd, Poole BH17 7NM, U.K. or from BDH Chemicals Ltd, Poole BH12 4NN, U.K.

\section{Fertility trials}

Hens of each species were randomly assigned to treatment groups with respect to their position in row and height of tier. Fowl hens received a single intravaginal insemination of $0.12 \mathrm{ml}$ diluted fowl semen $\left(160 \times 10^{6}\right.$ spermatozoa $)$ and turkey hens $0.1 \mathrm{ml}$ diluted turkey semen $\left(150 \times 10^{6}\right.$ spermatozoa). Fertilizing ability of aliquots of the same pooled semen sample was assessed under 2-3 different conditions of treatment.

The mean proportions of fertile eggs laid by hens for each treatment group during Days 2-8 (fowl) and 2-15 (turkey) after insemination were calculated.

Batches of eggs were held for 4 days before being placed in the incubator. Fertility of eggs was 
estimated by candling with an incandescent light between Days 4 and 8 of incubation. Eggs that appeared to be infertile were opened and examined for signs of fertility. Cracked, unincubated eggs were broken open and their blastoderms inspected for evidence of fertility as described by Kosin (1944).

\section{Glycolysis}

\section{Results}

Fowl and turkey semen diluted in buffer without added glucose and incubated anaerobically did not produce lactate and can therefore be considered to be free of glycolytic substrate. The spermatozoa of the two species had similar rates of oxygen consumption in the absence of glucose (Text-fig. 1a), presumably as a result of metabolism of endogenous substrates, but differed in their metabolism of glucose (Text-fig. la, b \& c).

Turkey spermatozoa only utilized significant amounts of glucose aerobically (Text-fig. 1b), increasing their oxygen consumption 2 -fold (Text-fig. 1a).
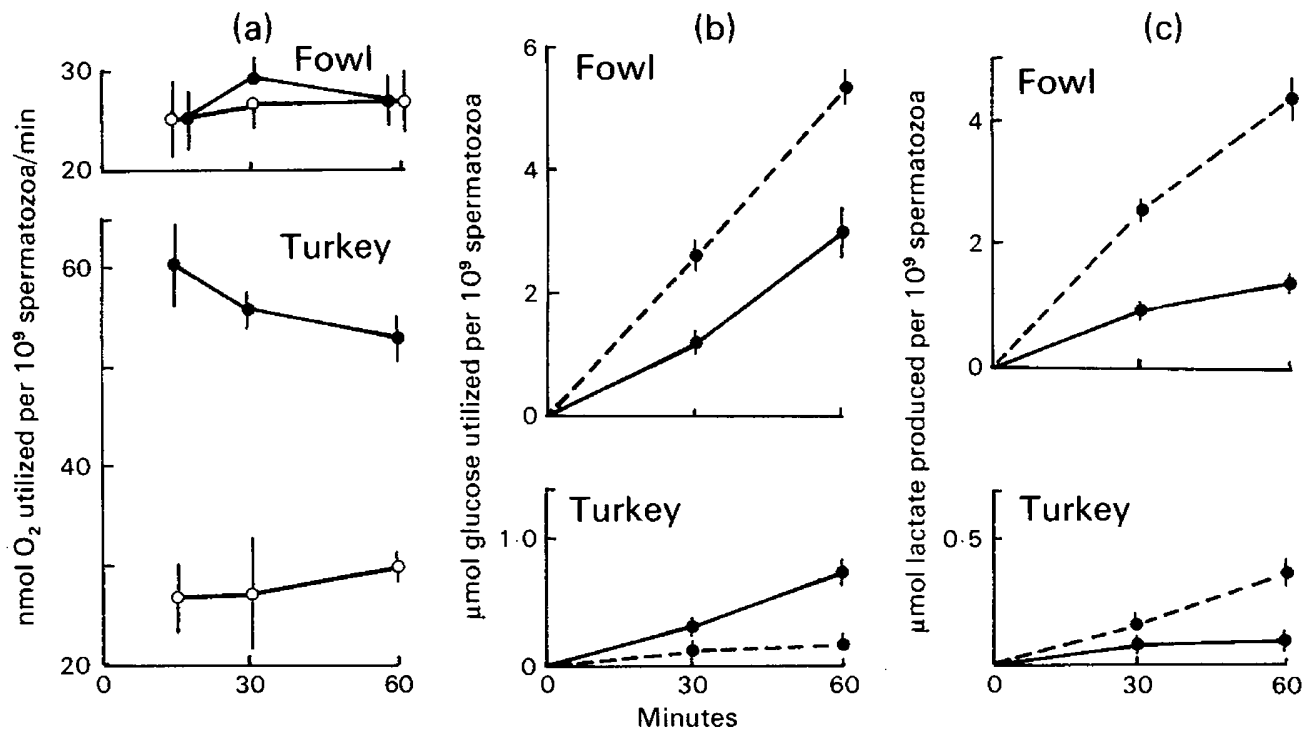

Text-fig. 1. Rate of (a) oxygen utilization, (b) glucose utilization and (c) lactate production by fowl and turkey spermatozoa at $40^{\circ} \mathrm{C}$ in vitro. Spermatozoa incubated in the presence of 10 mM-glucose; $O$, spermatozoa incubated in buffer only; ----, aerobic conditions; - - anaerobic conditions. Each point represents the mean \pm s.e.m. of $4(a, b)$ or 7 (c) determinations.

Fowl spermatozoa had a greater glycolytic capacity than turkey spermatozoa and, under anaerobic conditions, showed a 10 -fold higher rate of lactate production (Text-fig. 1c). In aerobic conditions lactate production continued at a decreased rate, showing a Pasteur effect (Text-fig. 1c), and the rate of oxygen consumption remained unchanged (Text-fig. 1a).

\section{Adenine nucleotides}

Under anaerobic conditions, without glucose, ATP levels in fowl and turkey spermatozoa were low after $15 \mathrm{~min}$ of incubation (Text-fig. 2b). In aerobic conditions, whether glucose was present or 
(a)

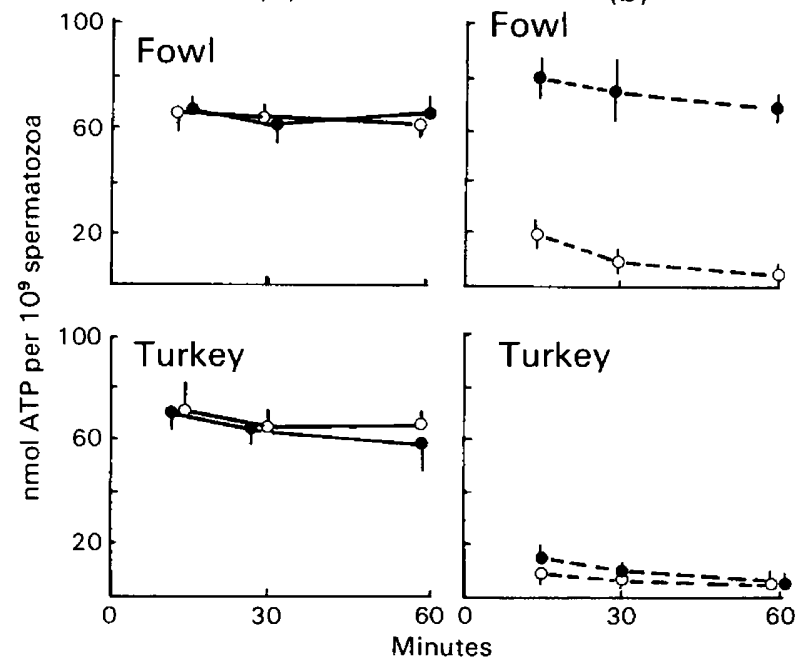

Text-fig. 2. ATP concentrations of fowl and turkey spermatozoa incubated under (a) aerobic and $(b)$ anaerobic conditions with $(O)$ and without $(O)$ the presence of $10 \mathrm{~mm}$-glucose. Values are mean \pm s.e.m. for 5 determinations.

not, high levels of ATP, equivalent to those of freshly-ejaculated spermatozoa (Table 1), were maintained over the incubation period in spermatozoa of both species (Text-fig. 2a). However, anaerobic glycolysis could only support 'fresh' levels of ATP in fowl spermatozoa. ATP concentrations in turkey spermatozoa fell as rapidly as under anaerobic conditions without glucose (Text-fig. 2b).

The fall in ATP levels in spermatozoa of both species incubated anaerobically without glucose was matched with a corresponding rise in ADP levels (Table 1) the transition from aerobic to anaerobic conditions resulting in a $90 \%$ fall in ATP :ADP ratios (Table 1). AMP levels in spermatozoa of both species also rose under anaerobic conditions, suggesting the presence of an active adenylate kinase.

Table 1. Adenine nucleotides in turkey and fowl spermatozoa

\begin{tabular}{|c|c|c|c|c|c|}
\hline \multirow[b]{2}{*}{ Semen incubation } & \multirow[b]{2}{*}{ Species } & \multicolumn{4}{|c|}{ Nucleotide conc. (nmol/109 spermatozoa) } \\
\hline & & ATP & ADP & AMP & ATP/ADP \\
\hline \multirow{2}{*}{ None (fresh) } & Turkey & $63 \cdot 6 \pm 8 \cdot 7(4)$ & $37 \cdot 0 \pm 2 \cdot 5(4)$ & $23 \cdot 7 \pm 7 \cdot 4(4)$ & 1.7 \\
\hline & Fowl & $57.8 \pm 9.9(9)$ & $30.3 \pm 4.8(9)$ & $29.8 \pm 8 \cdot 1(4)$ & 1.9 \\
\hline \multirow{2}{*}{ Aerobic } & Turkey & $75 \cdot 3 \pm 10.8(6)$ & $21 \cdot 1 \pm 4.6(6)$ & $17 \cdot 1 \pm 7.0(4)$ & $3 \cdot 6$ \\
\hline & Fowl & $81.5 \pm 9.0(6)$ & $26 \cdot 7 \pm 3 \cdot 3(6)$ & $33 \cdot 2 \pm 8.0(6)$ & $3 \cdot 1$ \\
\hline \multirow{2}{*}{ Anaerobic } & Turkey & $8 \cdot 8 \pm 2 \cdot 1(6)$ & $80.8 \pm 16.9(6)$ & $44.3 \pm 10.9(4)$ & $0 \cdot 11$ \\
\hline & Fowl & $17 \cdot 1 \pm 5 \cdot 1(6)$ & $86.8 \pm 13.7(6)$ & $72.4 \pm 16.2(6)$ & $0 \cdot 20$ \\
\hline
\end{tabular}

Incubations were performed for $30 \mathrm{~min}$ in buffer without glucose. Each result is the mean \pm s.e.m. of the number of estimations indicated in parentheses.

\section{Fertilization tests}

Under aerobic conditions without glucose, spermatozoa of both species maintained their fertilizing ability at $80-100 \%$ throughout the incubation period studied (Text-fig. 3a). Anaerobic incubation conditions affected turkey spermatozoa even when glucose was present in the medium but fowl spermatozoa were affected only when incubated without glucose (Text-fig. 3b). 
(a)

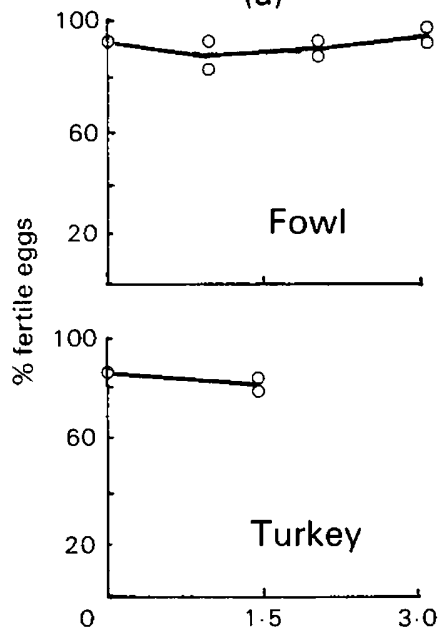

(b)
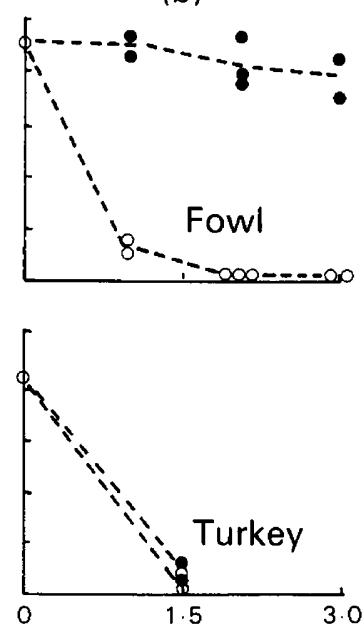

Hours

Text-fig. 3. Fertilizing capacity of fowl and turkey spermatozoa incubated under (a) aerobic or (b) anaerobic conditions with $(O)$ and without $(O) 10 \mathrm{~mm}$-glucose. Each point represents the fertile percentage of 36-52 eggs laid by a group of 6-8 fowl hens on Days 2-8 after insemination or of 74-88 eggs laid by a group of 9 or 10 turkey hens on Days 2-15 after insemination.

\section{Discussion}

These findings agree with previous work showing that fowl spermatozoa have a higher rate of anaerobic (Sexton, 1974) and aerobic (McIndoe \& Lake, 1973; Sexton, 1974) glycolysis than do turkey spermatozoa, although the Pasteur effect with spermatozoa of both species (Text-fig. 1c) and the increase in oxygen uptake of only turkey spermatozoa when glucose is added (Text-fig. 1a) have not been noted before. The present results show that certain aspects of the metabolism of fowl and turkey spermatozoa are remarkably similar. Thus, their rates of oxygen consumption when glucose is omitted from the medium are equivalent (Text-fig. 1a), as are their levels of ATP, ADP and AMP, whether from samples freshly ejaculated or incubated aerobically or anaerobically without glucose (Table 1). However, there are differences in their metabolism of glucose because fowl spermatozoa are glycolytically active at a rate sufficient to maintain ATP levels, whereas turkey spermatozoa use glucose significantly only under aerobic conditions, presumably by oxidizing derived metabolites. Fowl spermatozoa contain 20 times more lactate dehydrogenase activity than do turkey spermatozoa (McIndoe \& Lake, 1973) and this may be the reason for the observed metabolic differences.

Another difference in glycolytic metabolism between turkey and fowl spermatozoa is suggested by the present results. During anaerobic or aerobic incubation for $1 \mathrm{~h}$ with $10 \mathrm{~mm}$-glucose, the rate of lactate production by fowl spermatozoa falls considerably short of that expected from their glucose utilization (Text-fig. 1). This may be predicted since glucose is apparently metabolized to fructose by fowl spermatozoa (Lorenz, 1958), presumably because of some limitation in hexosephosphate metabolism. This would not be expected with turkey spermatozoa for which there appears to be a nearly stoichiometric relationship between glucose and lactate under anaerobic conditions.

The addition of glucose to respiring turkey spermatozoa doubled their rate of oxygen uptake and fowl spermatozoa will, aerobically, produce lactate from added glucose in addition to their aerobic metabolism of 'endogenous' substrates. However, neither of these increases in metabolic 
activity result in increased sperm ATP concentrations, indicating that ATP synthesis is uncoupled from respiration or ATP is utilized more rapidly.

The ATP and ADP levels and the ATP : ADP ratios of 'immediately' ejaculated fowl and turkey spermatozoa resemble, with allowances for a small time lapse $(3-4 \mathrm{sec})$ between collection and inactivation of 'fresh' samples, those of spermatozoa incubated aerobically in vitro but not those incubated anaerobically. It would therefore appear that sufficient oxygen is available within the ductus deferens to maintain ATP levels, in the absence of any glucose, by oxidation of as yet unidentified substrates.

The ATP concentrations of fowl and turkey spermatozoa in an 'optimal' energy state are about $70 \mathrm{nmol}(35 \mu \mathrm{g})$ per $10^{9}$ spermatozoa (Text-fig. 2). This is in marked disagreement with the previous reports of $5.5 \mu \mathrm{g}$ (Brackett \& Williams, 1967), $5 \mu \mathrm{g}$ (McIndoe \& Lake, 1969) and $2.5 \mu \mathrm{g}$ (Howarth, 1978) per $10^{9}$ fowl spermatozoa. However, Brackett \& Williams (1967) were unclear about the energy status of the spermatozoa used and McIndoe \& Lake (1969) and Howarth (1978) used an NADH-linked u.v. assay for ATP measurement in acid extracts of the spermatozoa; I have found this procedure unsatisfactory for the small amounts of ATP in such samples.

The fertilizability of fowl and turkey spermatozoa during incubation at $40^{\circ} \mathrm{C}$, at the times chosen in Text-fig. 3 and for several environmental conditions, exactly parallels their ability to maintain ATP levels (Text-fig. 2). When fowl and turkey semen samples are stored diluted in tubes at $5-10^{\circ} \mathrm{C}$, as is normal practice, they utilize oxygen at a rate greater than can be replenished by simple diffusion and soon become anaerobic (Wishart, 1981). It may then be predicted from the present results that fowl spermatozoa under such anaerobic conditions could produce sufficient ATP by glycolysis but that turkey spermatozoa could not. Aeration of semen during storage does produce considerable increases in the fertilizing ability of stored turkey semen and therefore in the length of time that turkey semen may be stored before artificial insemination (Wishart, 1981).

I thank Mrs Frances Ross for skilled assistance and Dr P. E. Lake for helpful discussion.

\section{References}

Brackett, B.G. \& Williams, W.L. (1967) ATP content of spermatozoa, semen and seminal plasma. Proc. Soc. exp. Biol. Med. 125, 1133-1136.

Burton, K. (1956) A study of the conditions and mechanisms of the diphenylamine reaction for the calorimetric estimation of deoxyribonucleic acid. Biochem. J. 62, 315-323.

Gutmann, I. \& Wahlefeld, A.W. (1974) L-(+)-Lactate. Determination with lactate dehydrogenase and NAD. In Methods of Enzymatic Analysis, pp. 14641468. Ed. H. U. Bergmeyer. Academic Press, New York.

Howarth, B., Jr (1978) Oxidation of U-14 C palmitic acid by cock spermatozoa. Poult. Sci. 57, 1751-1754.

Huggett, A. St.G. \& Nixon, D.E. (1957) Enzymatic determination of blood glucose. Biochem. J. 66, $12 \mathrm{P}$.

Kosin, I. L.(1944) Macro- and microscopical methods of detecting fertility in unincubated hens eggs. Poult. Sci. 23, 266-269.

Lake, P.E. (1957) Fowl semen as collected by the massage method. J. agric. Sci., Camb. 49, 120-126.

Lake, P.E. (1971) The male in reproduction. In Physiology and Biochemistry of the Domestic Fowl, pp. 14111442. Eds D. J. Bell \& B. M. Freeman. Academic Press, London.

Lake, P.E. \& Ravie, O. (1979) Effect on fertility of storing fowl semen for $24 \mathrm{~h}$ at $5^{\circ} \mathrm{C}$ in fluids of a different pH. J. Reprod. Fert. 57, 149-155.
Lake, P.E. \& Stewart, J.M. (1978) Artificial insemination in poultry. Bull. Min. Ag., Fish. Food, No. 213. Her Majesty's Stationery Office, London.

Iorenz, F.W. (1958) Carbohydrate metabolism of cock spermatozoa. Nature, Lond. 182, 397-398.

McIndoe, W.M. \& Lake, P.E. (1969) ATP and fowl spermatozoa. J. Reprod. Fert. 20, 360-362.

McIndoe, W.M. \& Lake, P.E. (1973) Aspects of energy metabolism of avian spermatozoa. J. Reprod. Fert. 35, 592-593.

Robinson, J. \& Cooper, J.M. (1970) Method of determining oxygen concentrations in biological media, suitable for calibration of the oxygen electrode. Analyt. Biochem. 33, 390-399.

Sexton, T.J. (1974) Oxidative and glycolytic activity of chicken and turkey spermatozoa. Comp. Biochem. Physiol. 48B, 59-65.

Strehler, B.J. (1974) Adenosine-5'-triphosphate and creatine phosphate. Determination with luciferase. In Methods of Enzymatic Analysis, pp. 2112-2126. Ed. H. U. Bergmeyer. Academic Press, New York.

Wishart, G.J. (1981) The effect of continuous aeration on the fertility of fowl and turkey semen stored above $0^{\circ}$ C. Br. Poult. Sci. 22, 445-450. 